\title{
List of addresses of the Jules Gonin Club's Members
}

1 Honorary Members

2 Passive Members

3 New Members elected in 1984

\section{Argentina}

Jose A. Badia

Junin 1616

1113 Buenos Aires

Ricardo Dodds

Rodriguez Pena 1386

Buenos Aires

Ricardo Hulsbus

Parera 164

1014 Buenos Aires

Enrique Malbran

Parera 164

1014 Buenos Aires

Hector H. Mocorrea

Av. Quintana 89-10-P.

Buenos Aires

Roberto Sampaolesi

Parana 1239 - $\mathbf{1}^{0}$ piso A

Buenos Aires
Alberto Urrets-Zavalia ${ }^{1} 1984$

Casilla de Correo 301

5000 Cordoba

\section{Australia}

J.D. Cairns

Suite 2, 14th Floor

15 Collins Street

Melbourne, Vic. 3000

G.W. Crock 21984

University of Melbourne

Dept of Ophthalmology

The Royal Victorian Eye and

Ear Hospital

32, Gisborne Street

Melbourne $\mathrm{C} 2$

Edgar J. Donaldson² 1984

The William Bland Centre

229 Macquarie Street

Sydney, NSW 2000

R.E. Hartley 21984

248 High Street

Penrith, NSW 2750

\section{Austria}

Suzanne Binder

Universitäts-Augenklinik

Spitalgasse 2

1090 Vienna

H. Freyler

Universitäts-Augenklinik

Spitalgasse 2

1090 Vienna

Hans Gnad

Universitäts-Augenklinik

Lange Gasse 72

1080 Vienna

Hans Hofmann²

Universitäts-Augenklinik

Landeskrankenhaus

Auenbruggerplatz 4

8036 Graz

Karl Hruby ${ }^{1}$

Universitäts-Augenklinik

Spitalgasse 2

1090 Vienna 
Erich Kutschera

Müllner Haupstrasse 48

5020 Salzburg

\section{Belgium}

Martine Brihaye-Van Geertruyden

Av. des Franciscains 98

1150 Brussels

E. Cambie

Gordunakaai 75

9000 Gent

Pierre Danis ${ }^{2}$

Av. Maurice 11

1050 Brussels

J.J. De Laey

Witte Leertouwerstraat 17

8000 Brugge

Hugo Verbraeken

Akademisch Ziekenhuis

De Pintelaan 135

9000 Gent

Marguerite Watillon

Clinique ophtalmologique

universitaire

Hôpital de Bavière

Bd de la Constitution 66

4020 Liège

\section{Brazil}

Sergio L. Cunha

Hospital das Clinicas

Caixa Postal 8091

Clinica Oftalmologica

São Paulo

\section{Canada}

Gordon S. Harris

2550 Willow Avenue

Vancouver V5Z 3N9

John C. Locke

Drummond Medical Bdg

Suite 1012

1414 Drummond Street

Montreal 107 P.Q. H3G 1W1

Clive B. Mortimer

Room 307, 5th floor

Toronto General Hospital

Toronto, Ontario M5G 1L7

Alain Rousseau

2790 Mont Royal

Ste Foy

Québec 10, G1W 2E2

Morris Shusterman

72, St Clair W.

Toronto M4V 1M7

\section{Chile}

T. Juan Verdaguer

Luis Thayer Ojeda 0115 - Of. 305

Santiago

\section{Colombia}

Jose I. Barraquer ${ }^{2}$

Apartado Aereo 90404

Bogota 8

Alvaro Rodriguez

Carrera 15, No. 76-60, Apto 201

Bogota D.E.

\section{Denmark}

Jens Edmund

Frederiksbergalle 60

1820 Copenhagen

Emil Frandsen ${ }^{2}$

Fredericiagade 17

6000 Kolding

Kim Frost-Larsen

Svalevej 10

2970 Hørsholm

Hans-Walther Larsen

Høyrups Allé 7, 5. tv.

2900 Hellerup

Erik Scherfig

Eye Dept. 2061, Rigshospitalet

Blegdamsvej 9

2100 Copenhagen

\section{Egypt}

Abdel-Latif Siam

3, Shagaret Eldorr Street

Zamalek

Cairo

Amin Zayed

10 Ismail El Mazny Street

Heliopolis

Cairo

\section{Finland}

Birgitta Knape 21984

Frederiksgaten $66 \mathrm{~B}$

00100 Helsingfors 


\section{France}

B. Algan

Groupe d'ophtalmologie

$M$. Barrès

36 , rue de la Ravinelle

54000 Nancy

Pierre Amalric

6 , rue St-Clair

81000 Albi

J.L. Arne

Hôpital Purpan

31000 Toulouse

Franck Bacin

C.H.U.

63000 Clermont-Ferrand

Pierre Bec

Centre Hospitalier Régional

Hôpital Rangueil

Service d'ophtalmologie

50 , Bd de Strasbourg

31052 Toulouse Cédex

Alain Bechetoille

4 , rue Larrey

Centre Hospitalier universitaire

49040 Angers Cédex

Pierre Bernard-Mettil

178, rue de Courcelles

75017 Paris

Mireille Bonnet

Clinique ophtalmologique

Universitaire B

Hôpital de la Croix Rousse

69317 Lyon Cédex 1

Paul Bregeat' 1984

9, rue Théodule-Ribot

75017 Paris
Henriette Chabat

26, Bd Bonne Nouvelle

75010 Paris

George Contantinides

Hôpital Régional

Service d'ophtalmologie

59000 Lille

Jacques Cordier ${ }^{2}$

18 , rue Marquette

54000 Nancy

Gabriel Coscas

203, rue de Vaugirard

75015 Paris

André Dubois-Poulsen ${ }^{1}$

14, rue Rémusat

75016 Paris

André Forest

23 bis, Av. Niel

75017 Paris

Pierre Francois

Centre Hospitalier Régional

Servie d'ophtalmologie

41, rue d'Artois

59000 Lille

Jean-Pierre Gehrard

27 , rue Goethe

67000 Strasbourg

Louis Guillaumat ${ }^{1}$

22, Place des Vosges

75004 Paris

Henry Hamard

1 , rue de Chazelles

75017 Paris
Jean Haut

Centre National d'ophtalmologie

des Quinze-Vingts

28, rue de Charenton

75571 Paris Cédex 12

François Hervouet ${ }^{2}$

16, Bd Guist'hau

44000 Nantes

Mme Sylvie Limon

Hôpital Avicenne

125 , rue Stalingrad

93000 Bobigny

Marcel Massin

5 , Villa Jocelyn

Square Lamartine

75016 Paris

Henri Miller

19, Bd de Beauséjour

75016 Paris

François Regnault

C.H.U. Bicêtre

78, rue du Général Leclerc

94270 Kremlin Bicêtre

J.P. Rouchy

11, Av. Elisée-Reclus

75007 Paris

Jacques-Blaise Saracco

C.H.U. Timone

Chemin de l'Armée d'Afrique

13385 Marseille Cédex 4

Henri Saraux

2, Square Alboni

75016 Paris

Gisèle Soubrane

78, rue du Général De Gaulle

94430 Chennevière 
Christian Zarrabi

6, Bd Wilson

06600 Antibes

\section{Germany}

E. Alexandridis

Universitäts-Augenklinik

Bergheimer Strasse 20

6900 Heidelberg

B. Von Barsewisch

Augenklinik Herzog Carl Theodor

Nymphenburger Str. 43

8 München 2

Wilhelm Boeke

Universitäts-Augenklinik

Hegewischstr. 2

23 Kiel

Ernst Custodis ${ }^{1}$

Himmelgeister Str. 69

4000 Düsseldorf 1

Wilhelm Doden

Universitäts-Augenklinik

Th. Stern-Kai 7

6000 Frankfurt-am-Main 70

Jörg Draeger

Hamburger Univ.-Augenklinik

2000 Hamburg

P. Gabel

Universitäts-Augenklinik

Mathildenstr. 8

8000 München

Jürgen Gaertner

Universiiitäts-Augenklinik

Langenbeckstrasse 1

6500 Mainz
Eva Gareis-Helferich

St-Marienkrankenhaus

Richard-Wagner-Strasse 14/16

6000 Frankfurt-am-Main

Edmund Gerke

Universitäts-Augenklinik

Hufelandstrasse 55

4300 Essen

Heinz Goertz

Augenklinik des

Kreiskrankenhaus Lüdenscheid

Philippstr. 2

5880 Lüdenscheid

Heinrich Harms ${ }^{2}$

Hohe Steige 16

7400 Tübingen

Ismail Hassouna

Am Urberg 34

4505 Bad Iburg

Klaus Heimann

Universitäts-Augenklinik

Joseph-Stelzmann-Str. 9

5000 Köln 41

Wolfgang Hoepping

Kantorie 97

4300 Essen 1

Henrich Honegger

Augenklinik

Med. Hochschule

Karl-Wiechert-Allee 9

3000 Hannover

Horst Hubner

Krankenhuas der Barmh. Brüder

5500 Trier

Jochen Kammann

St-Johannes Hospital

Johannes Str. 9-15

4600 Dortmund
Gerhard Keerl

Droste-Hülshoff-Str. 2

4000 Düsseldorf 30

Ingrid Kreissig

Ophthalmologie III

Universitäts-Augenklinik

Schleichstr. 12

7400 Tübingen

Peter Kroll ${ }^{3}$

Universitäts-Augenklinik

4400 Münster

Horst Laqua

Medizinische Hochschule Lübeck

Ratzeburger Allee 160

2400 Lübeck 1

Hans Liesenhoff

Augenklinik

Theodor Kutzer Ufer

6800 Mannheim 1

Hans Littmann ${ }^{2}$

Ziegelstr. 79

7920 Heidenheim

Otto-Erich Lund

Mathildenstr. 8

8000 München 2

Gunter Mackensen ${ }^{2}$

Universitäts-Augenklinik

7800 Freiburg in $\mathrm{Br}$.

Ulrich Mester ${ }^{3}$

Universitäts-Augenklinik Bonn

5300 Bonn-Venusberg

Gerd Meyer-Schwickerath ${ }^{1}$

Universitäts-Augenklinik

Hufelandstr. 55

4300 Essen 1 
Karl Mylius ${ }^{1}$

Chefarzt AK Barmbek i.R. agnesstr. 31

2000 Hamburg 60

Siegfried Niedermeier ${ }^{2}$

Augenklinik

Marianne-Rhodiusstr. 20

4150 Krefeld

Wolfgang Papst

Rübenkamp 148 - Augenklinik

2000 Hamburg 60

Helge Paulmann

Städtischeskrankenhaus

Augenklinik

Ostmerheimerstr. 200

5000 Köln 91

Friedrich Rentsch

Direktor der Augenklinik

Vincentius-Krankenhaus

Steinhäuserstr. 18

7500 Karlsruhe

Paul Schmitz-Valckenberg

Augenabteilung Ev. Stift St-Martin

5400 Koblenz

Manfred Spitznas

Universitäts-Augenklinik

Sigmund-Freud-Str. 25

5300 Bonn

Klaus Ullerich² 1984

Augenklinik der Städtischen

Kliniken

Beurhausstr. 40

4600 Dortmund

Theodor Waubke

Universitäts-Augenklinik

Hufelandstr. 55

4300 Essen
Achim Wessing

Universitäts-Augenklinik

Hufelandstr. 55

4300 Essen

Josef Wollensak

Augenklinik der Freien Universität

Krankenhaus Westend

Spandauer Damm 130

1 Berlin 19

German Democratic Republic

P. Lommatzsch

Karl-Marx-Universität

Direktor der Augenklinik

Liebigstr. 14

7010 Leipzig

Rudolf Sachsenweger ${ }^{2} 1984$

Augenklinik Leipzig

Liebigstrasse 14

7010 Leipzig

\section{Great Britain}

Norman H. Ashton 1

2, The Cloisters Westminster

Abbey

London SW1

Michael Bedford ${ }^{2}$

Lister House

11, Wimpole Street

London W1

Alan C. Bird

Moorfields Eye Hospital

City Road

London EC1V 2 PD

Rolf K. Blach

Lister House

11, Wimpole Street

London W1
Hector B. Chawla

The Royal Infirmary

Princess Alexandra Eye Pavillion

Chalmers Street

Edinburgh EH3 9HA

Anthony Hugh Chignell

44, Wimpole Street

London W1M 7DE

Ian Chisholm

Moorfields Eye Hospital

City Road

London EC1V 2PD

E.W.G. Davies

13, Upper Wimpole Street

London W1M 7TD

Andrew R. Elkington

Southampton Eye Hospital

Wilton Avenue

Southampton S09 4XW

Peter Fenton

Bare Farm House

North Boarthunt

Fareham, Hants P01 76JL

Timothy Hugh Ffytche

149, Harley Street

London W1N 2DE

Lorimer Fison ${ }^{1}$

62, Wimpole Street

London W1M 7DE

Wallace S. Foulds

Tennent Institute of Ophthal.

Western Infirmary

38, Church Street

Glasgow G11 6NT

Desmond Greaves

80, Harley Street

London W1N 1AE 
Peter Hamilton

149, Harley Street

London W1

James R. Hudson ${ }^{1}$

8, Upper Wimpole Street

London W1M 7DT

Barrie Jay ${ }^{2}$

10, Beltane Drive

London SW19 5JR

J.J. Kanski

Bay Lodge, Woodhill Avenue

Gerrards Cross

Bucks SL9 8DP

Satya B. Kapur

23, Farnham Close

Whetstone

London N20 9PV

Peter Leaver

Moorfields Eye Hospital

City Road

London EC1V 2PD

Margaret Eileen Leigh ${ }^{2}$

2, Park View Road

Ealing

London W5

David McLeod

Moorfields Eye Hospital

City Road

London EC1V 2PD

Brian Martin

Glebe House

5, Shaw Lane, Headingley

Leeds LS6 4DH

Hugh N. O'Donoghue

13, Kingsmere Road

London SW19 6PY
Michael J. Roper-Hall

38, Harborne Road

Birmingham B15 3HE

K. Rubinstein ${ }^{2} 1984$

10, Pritchatts Road

Edgbaston

Birmingham B15

John Scott

Addenbrooke's Hospital

Hills Road

Cambridge CB2 2QQ

C. Dee Shapland ${ }^{1}$

'Cornerways' Orley Farm Road

Harrow-on-Hill, Middx.

John Whitwell

Bagatelle

Burton Road

Branksome Park

Poole, Dorset

Greece

Spiros Pollalis

24, Kanapi Street

Athens 138

George Theodossiadis

Omirou 54

Athens 135

\section{Hungary}

D. Vörösmarthy

Budakeszi

Rakoczi ut. 4

2092 Budapest
Ireland

Philip E. Cleary

Regional Eye Department

Ardkeen Hospital

Waterford

F.D. McAuley

45, Upper Leeson Street

Dublin 4

Charles J. Maguire

25, Cleaver Avenue

Malone $\mathrm{Rd}$

Belfast BT9 6RX

Israel

Isaac C. Michaelson ${ }^{2}$

Hadassah Medical Center

P.O.B. 499

Jerusalem

Italy

Giorgio Bocci

Primario Oculista del Ospedale

Piazza Europa 20

12100 Cuneo

Mario R. Pannarale

Direttore della clinica Oculistica

Dell'University

Policlinico Umberto. $\mathrm{I}^{0}$

Rome 00197

Benedetto Strampelli

Circonvallazione Cornelia 65

Rome

Mario Valerio 21984

Borgonuovo 24

Milano 


\begin{tabular}{|c|c|c|}
\hline Japan & $\begin{array}{l}\text { Thore Lie Thomassen }{ }^{2} \\
\text { Rikshospitalet }\end{array}$ & $\begin{array}{l}\text { Joachim Barraquer }{ }^{2} \\
\text { Instituto Barraquer }\end{array}$ \\
\hline Hiroyuki Shimizu & Pilestredet 32 & Muntaner 314 \\
\hline Dpt of ophthalmology & Oslo 1 & Barcelona 6 \\
\hline \multicolumn{3}{|l|}{ Jichi Medical School } \\
\hline Minamikawachi-Machi & & Jose Casanovas ${ }^{1}$ \\
\hline Tochigi-Ken $329-04$ & Poland & $\begin{array}{l}\text { Lauria } 77,1 \\
\text { Barcelona } 9\end{array}$ \\
\hline Koichi Shimizu & Anna Bernardczykowa & \\
\hline Gunna University & Grochowska 28a & Alfredo Dominguez \\
\hline $\begin{array}{l}\text { School of Medicine } \\
\text { 3.39.15 Showa-Machi }\end{array}$ & Poznan $60-277$ & $\begin{array}{l}\text { Instituto Oftalmico Nacional } \\
\text { Velazquez } 75,4^{0}\end{array}$ \\
\hline \multirow[t]{2}{*}{ Maebashi 371} & $\begin{array}{l}\text { Witold J. Orlowski } \\
\text { Klinika Okulistyczna }\end{array}$ & Madrid 6 \\
\hline & Ul. Dluga $1 / 2$ & Carlos Heredia-Garcia \\
\hline Netherlands & 61.848 Poznan & $\begin{array}{l}\text { Paseo Manuel Girona 15, } \\
\text { Entresuelo }\end{array}$ \\
\hline A.F. Deutman & & Barcelona 34 \\
\hline University of Nijmegen & Portugal & \\
\hline Dept of Ophthalmology & & Francisco M. Honrubia-Lopez \\
\hline Ph. Van Leydenlaan 15 & Jose G.F. Cunha-Vaz & Urbanizacion Torrebarajas 29 \\
\hline \multirow[t]{2}{*}{ Nijmegen } & Clinica Oftalmologica & Carretera de Logrono \\
\hline & Hospitais da Universidade & Zaragoza \\
\hline J.A. Oosterhuis & Celas & \\
\hline c/o Oogheelkundige Kliniek & Coimbra & Jose L. Menezo \\
\hline Academisch Ziekenhuis & & Jorge Juan $6-4^{0}$ \\
\hline Rijnsburgerweg 10 & & Valencia 4 \\
\hline 2333 AL Leiden & South Africa & \\
\hline $\begin{array}{l}\text { Henk Parren } \\
\text { Herenlaan } 7 \\
\text { Zeist }\end{array}$ & $\begin{array}{l}\text { Ian L. Bradfield } \\
401 \text { Union Castle, Adderley Street } \\
\text { Cape Town } 8001\end{array}$ & $\begin{array}{l}\text { Simon A. Muinos } \\
\text { Clinica Barraquer } \\
\text { Muntaner } 314 \\
\text { Barcelona } 6\end{array}$ \\
\hline $\begin{array}{l}\text { Nicolas Schweitzer } \\
\text { Oogheelkundige Kliniek } \\
\text { Academisch Ziekenhuis } \\
\text { Groningen }\end{array}$ & $\begin{array}{l}\text { Gavin Douglas } \\
\text { P.O. Box } 4169 \\
\text { Johannesburg } 2000\end{array}$ & $\begin{array}{l}\text { Antonio Olivella-Casals } \\
\text { Escuela Oftalmologia } \\
\text { Av. Diagonal } 606 \\
\text { Barcelona } 21\end{array}$ \\
\hline Norway & $\begin{array}{l}\text { Spain } \\
\text { Alfredo Arruga }\end{array}$ & $\begin{array}{l}\text { Antonio Pinero-Carrion } \\
\text { Ciudad de Ronda } 4 \\
\text { Sevilla }\end{array}$ \\
\hline $\begin{array}{l}\text { Per Syrdalen } \\
\text { Rikshospitalet }\end{array}$ & $\begin{array}{l}\text { Pasaje Mendez-Vigo } 3 \\
\text { Barcelona }\end{array}$ & \\
\hline \multirow[t]{2}{*}{$\begin{array}{l}\text { Pilestredet } 32 \\
\text { Oslo } 1\end{array}$} & & Sweden \\
\hline & & $\begin{array}{l}\text { Peep Algvere } \\
\text { Karolinska Hospital } \\
10401 \text { Stockholm }\end{array}$ \\
\hline
\end{tabular}


Bertil Linder

Eye Clinic

Central Hospital

35185 Vexioc

Sven Oesterlin

University of Lund

Dpt of ophthlamology in Malmö

21401 Malmö

Ragnar Törnquist

15 , Stortorget

70211 Örebro

\section{Switzerland}

A.H. Alsaadi

Via Zorzi 17

6902 Paradiso

Jean Babel ${ }^{2}$

Clinique ophtalmologique

universitaire

22, rue Alc. Jentzer

1205 Genève

Roland Brückner ${ }^{2} 1984$

St Albanring 233

4052 Basel

Noëlle Chome ${ }^{2}$

Av. de Rumine 48

1005 Lausanne

Jean-François Cuendet ${ }^{2} 1984$

Av. de Rumine 31

1005 Lausanne

Basil Daicker

Universitäts-Augenklinik

Mittlere Strasse 91

4056 Basel
Nicolas Ducrey

Clinique ophtalmologique

universitaire

15, Av. de France

1004 Lausanne

Georg Eisner ${ }^{2}$

Universitäts-Augenklinik

Inselspital

Freiburgstrasse 8

3008 Bern

Franz Fankhauser ${ }^{2}$

Universitäts-Augenklinik

Inselspital

Freiburgstrasse 8

3008 Bern

Jürgen Faulborn ${ }^{3}$

Universitäts-Augenklinik

Mittlere Str. 91

4056 Basel

Claude Gailloud

Clinique ophtalmologique

universitaire

15, Av. de France

1004 Lausanne

Balder Gloor

Universitäts-Augenklinik

Mittlere Strasse 91

4056 Basel

Hans Goldmann ${ }^{1}$

Humboldstrasse 39

3013 Bern

Gabrielle Gonin ${ }^{1}$

Chemin de Rovéréaz 14

1012 Lausanne

Michel Gonvers

Clinique ophthalmologique

universitaire

15, Av. de France

1004 Lausanne
Alfred Huber ${ }^{2}$

Stadelhoferstrasse 42

8001 Zürich

Rüdolf Klöti ${ }^{1} 1984$

Universitäts-Augenklinik

Rämistrasse 100

8091 Zürich

Adolf Leuenberger

Augenklinik Kantonsspital

Buchserstrasse

5000 Aarau

Friedrich Rintelen ${ }^{2}$

Bruderholzrain 55

4059 Basel

Jean Rumpf ${ }^{2}$

Le Cotterd

1867 Ollon s/Aigle

Bernardo Streiff ${ }^{1}$

Av. Tissot 18

1006 Lausanne

Rüdolf Witmer

Universitäts-Augenklinik

Rämistrasse 100

8091 Zürich

\section{United States}

Thomas M. Aaberg

Dept of Ophthalmology

Med. College of Wisconsin

8700 W. Wisconsin Ave.

Milwaukee, WI 53226

Lloyd Aiello

Beethan Eye Unit

Joslin Clinic

1, Joslin Place

Boston, MA 02215 
William H. Annesley

Lankenau Medical Bdg

Lancaster Ave. and City Line

Philadelphia, PA 19151

Jose Berrocal

Oftalmologia

Hospital Mimiya

Santurce, Puerto Rico 00909

George Blankenship

Bascom Palmer Eye Institute

Dept of Ophthalmology

P.O. Box 016880

Miami, FL 33101

Isaak Boniuk

East Pavillion, Suite 17413

4949 Barnes Hospital Plaza

St Louis, MO 63110

Helmut Buettner

Mayo Clinic

Mayo Medical School

Rochester, MN 55901

Norman E. Byer

3400 West Lomita Bd

Torrance, CA 90505

John Clarkson

Bascom Palmer Eye Institute

P.O. Box 016880,

Miami, FL 33101

Gilbert W. Cleasby

Pacific Medical Center

P.O. Box 7999

San Francisco, CA 94120

David Cogan

4713 Trent Court

Chevy Chase, MD 20015
D. Jackson Coleman, M.D.

The New York Hospital

Cornell Medical Center

525 East 68 Street

New York, NY 10021

Victor T. Curtin

Bascom Palmer Eye Institute

1638 N.W. 10th Avenue

Miami, FL 33101

Matthew D. Davis

University of Wisconsin

Hospital \& Clinics

600 , Highland Ave.

Madison, WI 53792

A. Dellaporta

1101 Welch Road, Suite B-7

Palo Alto, CA 94304

Graham Dobbie

845 N. Michigan Ave, Suite 930 E

Chicago, IL 60611

Nicholas G. Douvas

4200 Gratiot Ave.

Port Huron, MI 48060

George Z. Dupont ${ }^{2}$

601 Dover Drive, Suite 3

Newport Beach, CA 92663

Guerry III Dupont ${ }^{2}$

Monument Avenue Eye Center

2015 Monument Ave.

Richmond, VA 23220

Robert M. Ellsworth² 1984

515 East, 71st Street

New York, NY 10021

William G. Everett

Everett \& Hurite Ophtalmic Ass.

Washington Plaza

1420 Center Avenue

Pittsburgh, PA 15219
Constance R. Fitzgerald

720 S.W. 2nd Avenue, Suite 306

Gainesville, FL 32601

Hal Mackenzie Freeman

Retina Associates

100, Charles River Plaza

Boston, MA 02114

Dennis B. Freilich

120 East 81th Street

New York, NY 10028

Morton Wallace Friedman

2233 Post Street

San Francisco, CA 94115

Wayne E. Fung

2351 Clay Street, Suite 414

San Francisco, CA 94115

John D. Gass

P.O. Box 016880

Bascom Palmer Eye Institute

Miami, FL 33101

Louis Girard ${ }^{2}$

4126 Southwest Freeway, Suite 500

Houston, TX 77027

Evangelos S. Gragoudas

Massachusetts Eye and

Ear Infirmary

243 Charles Street

Boston, MA 02114

Sohan Singh Hayreh

600 River Street

Iowa City, IA 52242

W.A.J. Van Heuven

UTHSC, Dept. of ophthalmology

7703 Floyd Curl Drive

San Antonio, TX 78284

George F. Hilton

3300 Webster Street, Suite 1110

Oakland, CA 94609 
Erwin Hultsch

University Hospitals \& Clinics

c/o Dept Ophthalmology

600 Highland Avenue

Madison, WI 53792

S. Rodman Irvine ${ }^{2}$

California College of Medicine

UCI Ophthalmology Group

19732 Mac Arthur Boulevard

Irvine, CA 92715

Glen P. Johnston

4989 Barnes Hospital Plaza

St. Louis, MO 63110

Otto H. Jungschaffer

12840 Riverside Drive

N. Hollywood, CA 91607

Allan E. Kreiger

Jules Stein Eye Institute

800 Westwood Plaza, UCLA

Los Angeles, CA 90024

Francis A. L'Esperance

1, E. 71th Street

New York, NY 10021

Harvey Lincoff

c/o New York Hospital

525 East, 68th Street

New York, NY 10021

Hunter L. Little

1225 Crane Street

Menlo Park, CA 94025

Robert Machemer

Dept of Ophthalmology

Duke University Medical School

Durham, NC 27710

A. Edward Maumenee ${ }^{1}$

Johns Hopkins University

The Wilmer Institute

Baltimore, MD 21205
P. Robb. McDonald²

Lankenau Medical Bdg

Lancaster Ave. and City Lane

Philadelphia, PA 19151

J.W. McMeel

Retina Associates

100 Charles River Plaza

Cambridge Street

Boston, MA 02114

Alice McPherson

Scurlock Tower, Suite 2200

6560 Fannin

Houston, TX 77030

Ronald G. Michels

108 Witherspoon RD

Baltimore, MD

Alfred J. Nadel

129 East, 69th Street

New York, NY 10021

Edward W.D. Norton ${ }^{1}$

Bascom Palmer Eye Institute

P.O. Box 016880

Miami, FL 33101

Edward Okun

4989 Barnes Hospital Plaza

St. Louis, MO 63110

Glen L. Oliver

2950 Interstate 45 , Suite 8

Huntsville, TX 77340

Karl C. Ossoinig

1216 Edingale Drive

Iowa City, IA 52240

L. Harrell Pierce

700 , Cathedral Street

Baltimore, MD 21201
Dohrmann K. Pischel ${ }^{1}$

490 Post Street

San Francisco, CA 94102

Charles D.J. Regan

Massachusetts Eye and

Ear Infirmary

243 Charles Street

Boston, MA 02114

Dennis Robertson

Mayo Clinic

Rochester, MN 55901

Melvin L. Rubin

Dept of Ophthalmology

College of Medicine, Med. Center

P.O. Box J. 284

Gainesville, FL 32160

Stephan Ryan

Estelle Dohemy Eye Foundation

Bdg

1355 San Pablo Street

Los Angeles, CA 90033

Felix Sabates

110 Rockhill Medical Bdg

6700 Troost

Kansas City, MO 64131

Charles Schepens ${ }^{1}$

100, Charles River Plaza

Boston, MA 02114

Donald Shafer

525 Park Avenue

New York, NY 10021

Anwar Shah

1035 Bellevue Avenue

Retina Institute

St. Louis, MO 63117 
Jerry Shields ${ }^{2} 1984$

Retina Service

Wills Eye Hospital

1601 Springs Garden Street

Philadelphia, PA 19130

Harold Spalter

635 West, 165th Street

New York, NY 10032

Bradley R. Straatsma

Jules Stein Eye Institute

800, Westwood Plaza, UCLA

Los Angeles, CA 90024

William Tasman

910 E. Willow Grove Avenue

Wyndmoor, PA 19118
Harvey E. Thorpe ${ }^{2}$

5820 Aylesbore Avenue

Pittsburgh, PA 15217

Rob. C. Watzke ${ }^{2}$

Dept. of Ophthalmology

University Hospitals

Iowa City, IA 52242

Paul C. Wetzig

616 So Tejon Street

P.O. Box 2170

Colorado Springs, CO 80902

Pat Wilkinson

McGee Eye Institute

Oklahoma Health Sciences Center

608 Stanton L. Young Bld

Oklahoma City, OK 73104
Uruguay

Raul Rodriguez-Barrios ${ }^{2}$

Clinique universitaire

ophtalmologique

Rio Negro 1222

Montevideo

\section{Yugoslavia}

Milan Blagojevic

Ocna Klinika

Dzordza Vasingtona 19

11000 Beograd 